commune we propose and evaluate improvements to the current system for tracking work-related injuries. We evaluate both passive and active approaches for capture sensitivity and the potential for collecting information on industries, occupations and populations at risk, injury types, causes, severity and burden.

Results Currently the Ministry of Health in Vietnam collects and publishes statistics on non-fatal injuries using hospital admission reports. A passive surveillance model which builds on the current system, but adds case reports for individuals treated at the commune health stations (CHS) and includes data from a newly designed additional injury log, would improve the capture of injuries and allow identification of occupations and populations at risk. However, with passive reporting the completion rate, accuracy and validity of the information collected are likely to be compromised. An active surveillance model, structurally similar to the passive approach, but gathering timely reports of injuries and including follow-up of injured individuals, would provide greater sensitivity of capture and case detail, while requiring significant resources. Active reporting in the Xuan Tien commune found counts of work-related injuries approximately 24 times higher than reported previously.

Conclusions We recommend that an extended passive surveillance approach be adopted in Vietnam to include hospital and community health station reporting. As health authorities become aware of counts or rates in specific communities which contribute disproportionately to the national burden, active surveillance in those communities might become a valuable extension to national surveillance.

\section{SNAKE BITE ENVENOMATION IN SAN, MALI}

${ }^{1} \mathrm{H}$ Hami, ${ }^{2}$ Coulibaly, ${ }^{2}$ Maïga, ${ }^{3}$ Mokhtari, ${ }^{4}$ Soulaymani-Bencheikh, ${ }^{3}$ Soulaymani. ${ }^{1}$ Kenitra, Morocco; ${ }^{2}$ Faculty of Medicine, Pharmacy and Dentistry, University of Bamako, Bamako, Mali; ${ }^{3}$ Ibn Tofail University, Kenitra, Morocco; ${ }^{4}$ Moroccan Poison Control Center, Rabat, Morocco

\subsection{6/oemed-2013-101717.71}

Objectives Envenoming resulting from snake bites is a serious public health problem in many regions of the world. The aim of this study is to describe the difficulties in the management of envenomation in the prefecture of San in Mali.

Methods A retrospective study of snake bites cases, recorded in the Health Reference Center of San (Ségou region) in 20012003, was conducted.

Results During the period of study, 88 victims were received and treated at the Health Reference Center of San. Of these, $42 \%$ were farmers and 6,8\% shepherds. Adults 15 years and over were most commonly concerned because of their socioprofessional activities (cattle breeding, gathering [3DOTS]). The majority $(50,6 \%$ of reported cases) were occurred during fieldwork, 24,7\% during nature walks and 9,4\% during picking. Snakes belong to the Viperidae family (Bitis arietans, Echis ocellatus) and the Elapidae family (Naja nigricolis). In 50,6\% of cases, the bite was on the lower limb, $48,2 \%$ on the upper limb and $1,2 \%$ on the trunk. According to data available, $28,4 \%$ of envenomated patients have benefited from antivenom administered intravenously. The average length of stay in hospital was 3 days, with a range of 12 hours to 11 days. Among the 29 cases for whom the evolution is known, 7 of them died. For other cases, the outcome was favourable with or without sequelae.
Conclusions Concerted action is needed to ensure adequate supplies of effective antivenom to develop systems that deliver high quality health care.

\section{MENTAL OUTCOME OF WORKERS 12 MONTHS AFTER OCCUPATIONAL INJURY}

${ }^{1} S$ C Shiao, ${ }^{2}$ Lin, ${ }^{3} \mathrm{Guo},{ }^{4}$ Liao, ${ }^{5} \mathrm{Guo},{ }^{6} \mathrm{Kuo},{ }^{7} \mathrm{Hu},{ }^{7} \mathrm{Hsu} .{ }^{1}$ National Taiwan University, Taipei, Taiwan; ${ }^{2}$ National Taiwan University/Occupational Medicine and Industrial Hygiene, Taipei, Taiwan; ${ }^{3}$ National Cheng Kung University/Institute of Behavioral Medicine, Taian, Taiwan; ${ }^{4}$ National Cheng Kung University Hospital/Department of Psychiatry, Taipei, Taiwan; ${ }^{5}$ National Taiwan University/Environmental and Occupational Medicine, Taipei, Taiwan; ${ }^{6}$ Chung-Shan Medical University/Department of Psychiatry, Taichung, Taiwan; ${ }^{7}$ Institute of Occupational Safety \& Health, Taipei, Taiwan

\subsection{6/oemed-2013-101717.72}

Objectives Workers hospitalised after occupational injury are at risk for psychiatric disorders. This study aimed to examine prevalence rates of both post-traumatic stress disorder (PTSD) and major depression at 12 months in workers experiencing different types of occupational injury in Taiwan.

Methods Workers sustaining occupational injury and hospitalised for 3 days or longer and received hospitalisation benefits from the Labour Insurance program were recruited in this study. A two-staged survey study was conducted. A self-reported questionnaire including the Brief Symptom Rating Scale (BSRS-50) and Post-traumatic Symptom Checklist (PTSC) was sent to workers at 12 months after injury. Those who scored high and suspected to have mental conditions were recruited for the second stage phone interview with a psychiatrist using the Mini-international Neuropsychiatric Interview (MINI).

Results A total of 1233 workers completed the questionnaire. Among them, 167 (13.5\%) scored high in either BSRS or PTSC and were invited for the MINI interview. A total of 106 (63.5\%) completed the phone interview. The estimated rates of PTSD, partial PTSD (PPTSD), major depression, comorbid PTSD/ PPTSD and major depression, and either PTSD/PPTSD or major depression were $3.4 \%, 1.8 \%, 2.0 \%, 2.0 \%$, and $5.2 \%$, respectively. The risk factors for high scores in either BSRS or PTSC were gender, education level, marriage status, loss of consciousness after occupational injury, injury affecting physical appearance, occupational injury experience before this event, traumatic life events before and after this injury, length of hospital stay, self-rated injury severity, and the worker's proportion of income contribution to the family.

Conclusions Occupational injury can cause long-term mental condition in the workers. The identified risk factors in this study may provide valuable information for developing preventive strategies.

\section{TARGETING PREVENTION POLICIES AND PRIORITIES FROM USING ROUTINE OCCUPATIONAL INJURIES STATISTICS; ARGENTINA, 2012}

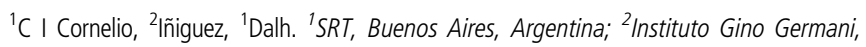
UBA, Buenos Aires, Argentina

\subsection{6/oemed-2013-101717.73}

Objective To identify from routine collected data in National worker's compensation database, sectors of the economy where workers are exposed to different risk levels, and to rank and prioritise groups to apply measures based on seriousness and coverage. 